Aus dem Fliegerärztlichen Institut der Abteilung für Flieger und Fliegerabwehr Leiter: PD Dr. K. WIEsINGER $\dagger$

\title{
Die Erscheinungen des Sauerstoffmangels, ein geschichtlicher Beitrag*
}

\section{Von Marco Mumenthaler}

Die Luftfahrtmedizin hat sich immer wieder mit dem Problem der Sauerstoffmangelerscheinungen in großen Höhen und mit deren Bekämpfung zu befassen. Aber auch bei der Besteigung der höchsten Berggipfel spielt der Sauerstoffmangel eine wesentliche Rolle. Während wir heute in der Sauerstoffatmung einen wirksamen Schutz gegen hypoxämische Erscheinungen besitzen, waren frühere Generationen wehrlos.

In dieser Studie soll ein Überblick über die Entwicklung unserer Kenntnisse auf diesem Gebiete gegeben werden. Es soll über die frühesten Schilderungen der Höhenkrankheit, über spätere Studien des Sauerstoffmangels und seiner Folgen auf Körper und Geist sowie über die Deutung und Bekämpfung dieser Symptome berichtet werden.

Naturgemäß waren es zunächst die Bergsteiger, denen die Wirkung der verdünnten Luft auffallen mußte. Schon die Sage schreibt der dünnen Höhenluft besondere Eigenschaften zu. So berichtet Aristoteles (erwähnt bei Boyle), daß die Besteiger des Olymps wegen der geringen Dichte jener Luft nur durch nasse Schwämme einatmen könnten. Fralichius beschreibt ebenfalls die dünne Luft auf den höchsten Karpathengipfeln : «ad summum usque montis pervenisset aerem adeo intempestum compariebat et subtilem».

Die Berge Europas waren jedoch nicht hoch genug, als daß auf ihren Gipfeln regelmäßig stärkere Symptome der Bergkrankheit aufgetreten wären. Erst mit der Entdeckung der neuen Welt und den kühnen Zügen der spanischen Conquistadoren über die Anden wurden die schweren Erscheinungen des Sauerstoffmangels bekannt. Der klassische Bericht des Paters Acosta schildert erstmals 1590 eindrücklich und genau die Bergkrankheit wie folgt:

* Herrn Major K. Wiesinger zugeeignet, der am 27. August 1955 den Fliegertod starb. 
«Hay en el Perú una sierra altísima, que llaman Pariacaca; yo habia oído decir esta mudanza que causaba, y iba preprado lo mejor que pude ... Y con toda mi preparacion, quando subí las escaleras, que llaman, que es lo mas alto de aquella sierra, cuasi súbito me dió una congoja tan mortal, que estuve con pensamientos de arrojarme de la cabalgadura en el suelo. Y porque anunque íbamos muchos, cada uno apresuraba el paso, sin aguardar compañero, por salir presto de aquel mal parage, solo me hallé con un Indio, al cual le rogué me ayudase á tener en la bestia. $\mathrm{Y}$ con esto luego tantas arcadas y vómitos, que pensé dar el alma ... llegué á echar sangre, de la violencia que el estómago sentía. Finalmente digo, que si aquello durara, entendiera ser cierto el morir, mas no duró sino obra de tres ó cuatro horas, hasta que bajamos bien abajo, y llegamos á temple mas conveniente, donde todos los compañeros, que serian catorce ó quince, estaban muy fatigados, algunos caminando pedian confesion pensando realmente morir. Otros se apeaban, y de vómitos y cámaras estaban perdidos : á algunos me dijeron, que les habia sucedido acabar la vida de aquel accidente. Otros ví yo, que se echaba en el suelo, y daba gritos del rabioso dolor que la habia causado la pasada de Pariacaca. Pero lo ordinario es no hacer daño de importancia, sino aquel fastidio y disgusto penoso que da mientras dura. $\mathrm{Y}$ no es solamente aquel paso de la sierra Pariacaca el que hace este efecto, sino toda aquella cordillera, que corre á la larga mas de quinientas leguas, y por donde quiera que se pase, se siente aquella extraña destemplaza, aunque en unas partes mas que en otras...»

Pater Acosta wurde also beim Übergang über einen hohen Gebirgszug in Peru, der Pariacaca, von einem derartigen Unwohlsein befallen, daß er sich am liebsten von seinem Reittier auf den Boden geworfen hätte. Jeder einzelne seiner Reisegefährten kümmerte sich nicht mehr um die anderen und beeilte sich nur, die unwirtliche Gegend so rasch als möglich zu verlassen. Der begleitende Indianer mußte den Reiter auf dem Tiere stützen. Das Erbrechen - auch von Blut - war so heftig, daß Acosta meinte sterben zu müssen. Das Ganze aber dauerte nur drei bis vier Stunden, bis die Reisenden in tiefere Regionen gelangten. Alle waren sehr erschöpft, einzelne wollten auch beichten, da sie dachten, sterben zu müssen. Andere stießen wütende Schmerzschreie aus. Im allgemeinen aber verursachten diese Störungen - die auch in anderen Teilen jener Gebirge auftraten - keinen ernstlichen Dauerschaden.

Die Eingeborenen versuchten sich gegen die Erscheinungen der Bergkrankheit - wie dieses Unwohlsein von Acosta erstmals genannt wurde dadurch zu schützen, daß sie sich Gehörgänge, Nase und Mund verstopften und besonders auch die Magengegend mit warmen Kleidungsstücken bedeckten. Übrigens litten auch die Tiere unter der Bergkrankheit:

«... todo el remedio (y lo es muy grande) que hallan es, en taparse cuanto pueden oídos, y narices, y boca, y abrigarse de ropa especialmente el estómago ... y no solo los hombres sienten aquella congoja, pero tambien las bestias ... » 
Die Schutzmaßnahmen der Eingeborenen gegen das Eindringen der schädlichen Luft in ihre Körperöffnungen, aber auch weil jene Bergzüge zu den höchsten der Erde gehören, brachten Acosta zur Überzeugung, daß in der Eigenheit jener dünnen Luft die Ursache der verspürten Störungen liegen müsse. Er meint wörtlich:

«Ich bin der Auffassung, daß jene Gegend zu den höchsten der Erde gehört ... und so bin ich überzeugt, daß die Luft daselbst so dünn und schwach ist, daß sie nicht für die menschliche Atmung geeignet ist, denn hierfür müßte sie dichter und wärmer sein. Das ist - so glaube ich - der Grund, warum der Magen so sehr angegriffen und der ganze Mensch so sehr verändert wird»*. (Übersetzt vom Referenten)

Auch spätere Beschreiber der Bergkrankheit im Hochlande Perus, z.B. UlloA, schildern sie auf ähnliche Weise:

Discours cinquième, Tome I «... c'est le Maréo de la Puna ... C'est une maladie toute semblable à celle qu'on éprouve en se mettant en mer : elle en présente aussi tous les symptômes, et suit le même ordre. La tête tourne, on sent de très grandes chaleurs, et il survient des nausées pénibles, suivies de vomissements bilieux. Les forces tombent, le corps s'abbat : la fièvre s'y joint, et le seul soulagement qu'on y trouve, c'est de vomir. Certains sujets y sont même si avattus, qu'ils donneroient de l'inquiétude, si l'on n'étoit certains que ce n'est autre chose que ce maréo. Cela dure ordinairement un jour ou deux, après quoi la santé se rétablit. Cette incommodité est plus ou moins considérable selon la disposition naturelle des personnes, mais peu y échappent ... Ils sentent en marchant une fatigue, comme soffocante et très pénible, qui les oblige de se reposer longtemps. Cela leur arrive même dans le plat pays.»

Wie bei der Seekrankheit verspürt der Reisende Schwindel, Wallungen, Brechreiz und Erbrechen. Eine erdrückende Müdigkeit zwingt ihn zum häufigen Ausruhen. Auch Ulloa - wie schon Pater Acosta - führt diese Störungen auf eine Eigenschaft der Luft zurück, sei es deren geringe Dichte, sei es eine andere unbekannte Eigenschaft:

«On ne peut sans doute attribuer cet accident au froid, car s'il en étoit la seule cause, cette maladie seroit commune dans tous les pays froids. Il faut donc que cela vienne de la qualité de l'air, soit en conséquence de sa légéreté, soit de toute autre qualité que nous n'y connoissons pas.»

Ähnliche Erscheinungen, aber in geringerer Ausprägung, wurden von Borellus im 17. Jahrhundert auf dem Ätna beobachtet:

* Kap. III : «Tengo para mi, que aquel parage es uno de los lugares de la tierra que hay en el mundo mas alto ... y así me persuado que el elemento de el aire está allí tan sutil y delicado, que no se proporciona á la respiracion humana, que le requiere mas grueso y mas templado; y esa creo es la causa de alterar tan fuertemente el estómago, y descomponer todo el sugeto.» 
Lib. II, Cap. 123 : «Inter admiranda, quae in vertice Montis Aetnaei anno 1671 observavi, fuit effectus inopinatus, productus ab excedente raritate illius aeris : ibi enim quilibet mediocris motus, ascendendo per salebrosas illas rupes, tam grandem lassitudinem producebat, ut etiam juvenes robusti, atque rustici saepè cogerentur sedendo quiescere, et frequenti anhelitu vires reficere. Notavi postea, quòd talis passio non producebatur à nimia subtilitate illius aeris, nec ab aliqua prava ejus qualitate; nam sedendo, aut equitando eundem aerem excipiebamus, et tamen benè respirationem exercebamus absque anhelitu, ac in infimis locis marinis respirari solet.»

Es fiel dem neapolitanischen Gelehrten also auf, daß selbst eine nur mäBige Anstrengung, wie z.B. das Hinaufsteigen am Berghang, genügte, um sogar junge und kräftige Leute außer Atem zu bringen. Sitzen, oder aber das Reiten, bewirkten keine Veränderung der Atmung. Daraus zog Borellus den Schluß, daß nicht allein die geringe Dichte jener Luft oder eine schädliche Eigenschaft derselben die Ursache für eine Atemstörung sein könne. Er äußerte als erster die Ansicht, daß die körperliche Arbeit als wichtiger Teilfaktor der unangenehmen Erscheinungen in der Höhenluft betrachtet werden müsse:

«Itaque ostendendum solummodo restat, quòd idem motus musculorum, qui in communi aere crasso tolerabili difficultate, et non molesta lassitudine absolvitur, postea in aere rarissimo non sine ingenti labore et lassitudine perfici potest.»

Borellus ist der Auffassung, daß die Luft in der Brust durch ihre elastische Kraft, durch Druck auf «Gas- und Blutgefäße», die Muskeltätigkeit unterstütze. Bei zu geringer Dichte der Luft jedoch, werde die Anstrengung der Muskeln weniger wirksam gestützt, und es müsse die gleiche Arbeit mit größerer Anstrengung geleistet werden:

«Modò, quia aer, in pecto cohibitus, sua vi elastica comprimendo vasa sprituosa et sanguinea, adjuvat conatum musculorum, ut dictum est; ergo, quando aer est rarissimus (licèt aequè à thorace comprimatur, ac aer densus) minùs validè elidet vasa; et ideò minùs adjuvabit conatum musculorum. Quare in aere rarissimo vis motiva musculorum imminuta erit ab defectum adjumenti, quod afferretur à vi elastica aeris condensati. Igitur in aere rarissimo eadem resistentia, à vi motiva diminuta, majori labore ac lassitudine sustinebitur.»

Übrigens hat Borellus bereits erkannt, daß auch zu große Dichte der Luft jedes Lebewesen ersticken und töten kann:

«Sicuti aëris superflua raritas subitò decubitum mortiferum inducit, sic quoque condensatio et crassities excessiva ejusdem animalia suffocat et extinguit.»

In späteren Jahren wurden von Forschungsreisenden und Bergsteigern immer wieder die Erscheinungen der Bergkrankheit beschrieben. Besonders traten sie in den Anden Südamerikas auf (Döppig, Fitz-Gerald, 
Güssfeldt, D'Orbigny, Whymper) und im Gebirge Asiens (Conways, Moorcroft, Schlagintweit). Klassisch ist die Beschreibung des Schweizers J. J. Tschudi, der 1838-1842 Peru durchstreifte. In seinem Reisebericht schildert er die allgemeinen Erscheinungen der Bergkrankheit mehrfach:

2. Buch, Kap. II, S. $67 \mathrm{ff}$ : «Die ersten Anzeichen der Veta erscheinen in der Regel auf einer Höhe von 12,600 Fuß ü. M., und bestehen in Schwindel, Ohrensausen und Trübesehen, wozu sich bald Kopfschmerzen und Übelkeit gesellen. Auch wenn man zu Pferde sitzt, treten häufig diese Erscheinungen auf, mit verdoppelter Stärke aber, wenn man zu Fuße bergan geht. Je höher man steigt, desto intensiver werden sie und vermehren sich durch Müdigkeit in den Oberschenkeln, die sich bis zur Unmöglichkeit des Gehens steigert, durch eine äußerst beengte Respiration und ein heftiges Herzklopfen. Eine vollkommene Ruhe vermindert zwar für Augenblicke diese Symptome, die aber bei der fortgesetzten Bewegung mit erneuter Heftigkeit wieder hervortreten und dann oft von Ohnmachten und heftigem Erbrechen begleitet sind. Die Capillargefäße der Bindehaut der Augen, die der Nase und der Lippen bersten und das Blut tritt tropfenweise aus ihnen hervor. Die nämlichen Erscheinungen zeigen sich auch auf den Schleimhäuten der Respirationsorgane und des Darmkanales. Blutspeien und blutige Diarrhöen sind daher häufige Begleiter der intensiven Veta.

Annäherungsweise kann man das Gefühl bei diesem Übel der Seekrankheit vergleichen (daher auch der Name Marreo), nur daß bei dieser die so sehr beängstigenden Atmungsbeschwerden fehlen. Nicht selten ist die Heftigkeit dieses Übels so groß, daß es dem Reisenden das Leben kostet... Sehr viel hängt von der Individualität und der Gewohnheit ab. Alle Küstenbewohner und Europäer, die zum erstenmale die hohe Cordillera passiren, leiden an dieser Krankheit, die bei nicht vollblütigen, gesunden Personen in der Regel schnell vorübergeht, bei schwächlichen, nervösen, Brust- oder Herzkranken, auch bei plethorischen und sehr fetten Individuen, einen sehr hohen Grad erreicht...

... Bei einem längeren Aufenthalte in diesen hohen Regionen gewöhnt sich der Organismus leicht an diese verdünnte Luft.»

Aber nicht nur die Menschen, sondern auch die Tiere sind der Bergkrankheit unterworfen, und die Eingeborenen wenden oft drastische Mittel dagegen an:

«Schon von Cacray an zeigt sich bei den der Gebirgsreisen ungewohnten Pferden die Wirkung des verminderten Luftdruckes, die sogenannte Veta. Sie fangen an langsam zu gehen, halten häufig stille, zittern am ganzen Leibe und stürzen zusammen. Je höher sie steigen, desto heftiger zittern sie, desto häufiger fallen sie um. Wenn man sie dann nicht absattelt, ruhen läßt und auf alle mögliche Weise schont, so gehen sie zugrunde. Einem so befallenen Thiere lassen die Arrieros an vier Stellen Blut ausfließen: nämlich an der Schwanzspitze, am Gaumen und an beiden Ohren. Oft schneiden sie ihm die Ohren und den Schwanz zur Hälfte ab, zuweilen die erstern glatt am Kopfe weg und schlitzen ihm die Nasenlöcher mehrere Zoll weit auf. Dieses letztere Mittel scheint mir allein von einigem Nutzen zu sein, da diese Thiere durch die gespaltenen weit geöffneten Nasenlöcher eine 
größere Menge Luft schöpfen können. Als Präservativ gegen die Veta wird ihnen zerstampfter Knoblauch in die Nasenlöcher gestrichen. Die Maulthiere und Esel sind der Veta weniger unterworfen als die Pferde, wahrscheinlich weil sie ruhiger steigen. Die in der Sierra gebornen Thiere sind fast ganz frei von diesem Übel.»

Die Schilderung seiner selbst erlebten, schweren Bergkrankheit ist äuBerst plastisch. Wir finden hier auch erstmals eine Andeutung, daß die Wirkung der Bergkrankheit nicht nur auf den Körper geht, sondern auch «moralisch» den Menschen schwächt und ihm den Willen raubt:

2. Buch, Kapitel 5, S. 152: «rüstig stieg ich bergan, doch begann ich auch alsobald den verderblichen Einfluß des verminderten Luftdruckes in diesen Höhen zu fühlen und bei jedem Schritte ergriff mich ein früher nie empfundenes Unbehagen. Ich mußte stillestehen, um Luft zu schöpfen, aber ich fand sie kaum. Ich versuchte zu gehen, aber eine unbeschreibliche Angst bemächtigte sich meiner. Hörbar klopfte das Herz gegen die Rippen, der Athem war kurz und abgebrochen, eine Welt lag mir auf der Brust. Die Lippen wurden blau, aufgedunsen und barsten. Die feinen aufgeschwollenen Capillargefäße der Augenlider rissen und tropfenweise drang das Blut heraus. In gleichem Maße verminderten sich die Sinnesthätigkeiten, ich sah und hörte und fühlte nichts mehr, ein dunkelgrauer Nebel schwamm vor meinen Augen, oft tief geröthet, bis ihnen eine blutige Thräne entquoll. Jetzt fühlte ich mich verwoben in den Kampf um Leben und Tod, den ich vorher in der Natur ahnte. Mein Kopf schwindelte, die Sinne schwanden, und zitternd mußte ich mich auf die Erde niederlegen. Wahrlich, wenn mich hundert Fuß höher die köstlichsten Güter der Erde oder die Glorie der Ewigkeit erwartet hätten, es wäre mir physisch und moralisch unmöglich gewesen auch nur die Hand nach ihnen auszustrecken.»

Güßfeldt hat ebenfalls diese Schwächung der Willensimpulse bemerkt und sagt: «So glichen wir mehr wandelnden Duldern als himmelerstürmenden Titanen.»

Ein anderer Schweizer, der Zürcher Arzt Conrad Meyer-Ahrens, widmete der Bergkrankheit 1854 erstmals eine Monographie. Seine ausgezeichnete Schilderung der vielfältigen Symptome möchten wir hier wiedergeben:

S. 100: «Die wesentlichsten, d.h. die am gewöhnlichsten vorkommenden Erscheinungen der Bergkrankheit, sind bei den Menschen: Ekel, Abneigung gegen Speisen, meist auch gegen den Wein (doch hat man auch von alle dem das Gegentheil beobachtet) starker Durst (in der Regel nach Wasser, das am meisten erquickt), UUbelkeiten mit Erbrechen. Beschleunigtes, keuchendes Athmen, beschleunigter Kreislauf, Pulsiren großer Arterien, z.B. der Schläfenarterien, Herzklopfen, heftige Oppression, Erstickungsangst, Schwindel, heftige Kopfschmerzen, Anwandlungen von Ohnmacht, unbezwingbare Schläfrigkeit, nicht erquickender, sondern durch Beklemmung gestörter Schlaf. Endlich außerordentliche, wahrhaft fabelhafte Erlahmung der Muskeln. Diese Erscheinungen sind - freilich nicht immer in vollständiger Combination - allenthalben, wo die Bergkrankheit vorkommt, beobachtet worden, und daher als wirklich wesentliche Erscheinungen zu betrachten. Es 
gibt daher sicherlich nur eine Art von Bergkrankheit, und jede Unterscheidung in mehrere Arten ist unstatthaft. Bei den Thieren, d.h. den Lastthieren, hat man ebenfalls allgemein beschwerliches Athmen und große Erlahmung der Muskeln beobachtet... Außer den bereits genannten hie und da noch andere Erscheinungen, welche in dieselbe Kategorie gehören, aber weniger allgemein beobachtet werden, als z.B. Nierenblutungen, Darmblutungen (auch bei Thieren), blutiges Erbrechen, Lungenblutungen, Blutungen aus den Lippen, der Haut, Abstumpfung der Sinnes- und Geistesthätigkeiten, oder Neigung zum Zorn, zur Ungeduld, regere geistige Thätigkeit, das Gefühl einer besonderen Leichtigkeit usw.»

Später erfuhr die Bergkrankheit durch Payot, v. Schrötter, KronECKER, durch Durig et al. sowie durch Zuntz und Mitarbeiter erneut ausführliche Darstellungen. Die letzteren Autoren, die sich auf eigene Erfahrung anläßlich einer Monte-Rosa-Expedition stützten, schilderten nicht nur die körperlichen Symptome der Bergkrankheit, sondern studierten auch besonders deren Wirkung auf das psychische Verhalten.

S. 424: «Gerade die Bergführer wissen von derartigen Reizzuständen ihrer Schutzbefohlenen zu berichten, wenn die Erregung sich auf dem Marsch in übermäßiger Angst oder auch in Überschätzung der eigenen Kraft und in Streitsucht zu erkennen gibt. Dann kostet es die ganze Überredungskunst des Führers, Unbesonnenheiten zu vermeiden oder den geschwächten Willen zu stärken. So erinnert das Verhalten der vom Hochgebirgsklima stark Angegriffenen an das des launenhaften und willensschwachen Neurasthenikers, dessen labiles Empfindungsleben den Einflüssen der Außenwelt wie seinen Organgefühlen schrankenlos ausgesetzt ist.

In großen Höhen kann sich die Erregung bis zu wirklichen Delirien steigern. Kräftige, berggewandte und schrittsichere Touristen taumeln auf unschwierigen Stellen wie Betrunkene, andere beklagen in bewegten Worten, daß sie sich so weit hinaufgewagt haben. Um jeden Preis wollen sie wieder in tiefere Regionen und lassen gern alle ihre Vorräte zurück, um nur schneller bergabsteigen zu können.»

Das Studium der Höhenkrankheit erfuhr im letzten Jahrhundert nicht nur durch das erwachende Interesse am Bergsteigen und durch die später zu besprechenden Ballonfahrten einen Aufschwung. Auch das Anlegen von Bergbahnen und die Ausbeutung hochgelegener Erzminen in den südamerikanischen Anden spornten zum Studium dieses Problems an. So verfaßte Kronecker 1903 seine Monographie über die Bergkrankheit im Auftrag des Komitees der Jungfraubahn (3454 m). Auch auf Pikes Peak in Colorado (4331 m) wo die höchste Bergbahn der USA hinaufführt, wurden sorgfältige physiologische Beobachtungen durch Douglas, Haldane und andere angestellt. Hier sowie auf der höchsten Bahn der Welt in Peru (4798 m) zeigte es sich, daß bei absoluter Ruhe kaum Störungen auftraten, während bei der kleinsten Anstrengung schon sich Beschwerden bemerkbar 
machten (Zuntz, 1906). Auch in den Erzminen von Cerro de Pasco (4400 m), der höchsten Stadt der Erde, sind die Erscheinungen der Bergkrankheit deutlich, ebenso aber die allmähliche Anpassung (Döppig).

Aber nicht nur Forscher vergangener Jahrzehnte hatten gegen die Bergkrankheit anzukämpfen, auch die Bergbesteigungen der neuesten Zeit boten in dieser Beziehung mannigfaltige Probleme (Оudot, Pfisterer). Ohne Sauerstoffatmung wären die Spitzenleistungen im Himalayagebiet nicht möglich gewesen. So hat z.B. schon die englische Mount-Everest Expedition unter BRUGE 1922 die Bedeutung der Sauerstoffatmung erkannt. Es heißt im Bericht der Expedition z.B.:

«.. The one factor, which renders the ascent so difficult is the want of oxygen in the air. Provide the oxygen and the ascent would be made at once... There is little doubt that it was the use of oxygen which saved our lifes during the second night in our high camp.»

Am Rande dieses historischen Überblickes über die Bergkrankheit sei auch kurz auf die frühesten Ansichten über die therapeutische Wirkung der Höhenluft hingewiesen (zum Teil zitiert nach Zuntz). Nach der Sage soll Apollo den schwächlichen Sohn Asklepios zum Berge Pelion gebracht haben, um ihn dort aufziehen zu lassen. GALEN empfiehlt für Lungenkranke mittlere Berghöhen. Antrulus (3. bis 4. Jahrhundert) sagt, daß die hochliegenden Orte so gut durchlüftet seien, daß sie nützlich für alle Krankheiten der Brust, sowie für diejenigen des Kopfes und aller Sinnesorgane seien. Marco Polo berichtet in seiner Reiseschilderung, daß im Hochland von Baddachschan die Bewohner bei Erkrankungen sich auf die Berge hinaufbegeben, wo sie in einigen Tagen ihre Gesundheit wieder erhalten.

Ulloa schildert 1764 die günstige Wirkung der Höhenluft auf Asthmatiker:

S. 120: «Cette légéreté de l'air devient favorable aux asthmatiques devenus tels dans un air plus épais. Cet asthme y est connu sous le nom de ahogos ou suffocation. Il y est même assez commun. C'est pourquoi ceux qui essont attaqués dans les vasses contrées, se rendent dans les hautes, quoiqu'ils n'y guérissent pas entièrement, ils y vivent cependant sans peine. Ceux au contraire qui sont devenus tels dans les hauts pays, se trouvent bien dans les bas. Ainsi le changement d'air devient un soulagement assuré dans cette expèce d'incommodité. La médecine pourroit tirer parti de ces expériences, en envoyant les malades d'une contrée dans une autre, quoiqu'il n'y eût pas ailleurs une aussi grande différence dans l'élévation des terreins»

Jourdanet, der selber Arzt war und während 19 Jahren in Mexico lebte, sagt u. a.: 
S. 296: «De toutes les maladies, en effet, qui empruntent un haut intérêt aux considérations prophylactiques dont le climat qui nous occupe puisse être la base, la tuberculisation pulmonaire doit sans nul doute s'inscrire au premier rang.»

Er beschreibt übrigens auch den Höhenschwindel und belegt denselben auch mit dem medizinischen Namen: «Cerebro-anémie vertigineuse aiguë».

Näheres zu diesem Thema vgl. auch Zuntz, Küchenmeister.

Überall dort, wo die Bergkrankheit vorkommt, haben die Einheimischen eine besondere Bezeichnung für diese Erscheinungen, die zum Teil auch schon auf die vermuteten Ursachen derselben hindeutet (vgl. Kronecker). Die Bezeichnung «Bergkrankheit» selber soll auf Acosta zurückgehen, und diesem Ausdruck entsprechen das französische «mal des montagnes», das englische «mountain sickness», das italienische «mal di montagna» und das spanische «mal de las montañas». Wegen der Ähnlichkeit mit der Seekrankheit bezeichnete Ulloa die Bergkrankheit als «mareo de la Puna». Auch «Puna» allein - das peruanische Hochland - wurde für die Bergkrankheit gebraucht. Die indianische Bevölkerung der Anden nenne sie «Cnunnus» durch Kälte erhärtet - oder «Sorroche». Dieses Wort soll - nach MiddenDORF - von einer falschen Aussprache des Keshua-Wortes Sorrojchi herrühren, was Schwefelkies bedeute. Dieselbe Deutung der Bergkrankheit ist im spanischen Ausdruck «Veta» enthalten, d.h. Erzgang. Eine andere spanische Bezeichnung lautet «Quebranta huezzos», was so viel wie «ermattende Mühsal» bedeutet. Wie die Gebrüder Schlagintweit berichten, wird die Bergkrankheit in Hochasien «Bitsch» oder «Bischki Hana», auch «Kharab Hana», d.h. böse giftige Luft genannt, oder als «Serân», d.h. giftiger Wind - bezeichnet. In Tibet heißt sie «Bies» oder «Mundara». Auf Borneo wird ein Unwohlsein auf den Bergen «Ikak»genannt.

Während zunächst nur die Besteigung hoher Berge den Menschen die Höhenkrankheit erleben ließ, waren später auch die Ballonfahrer der Hypoxämie ausgesetzt. Die Brüder Montgolfier, die 1783 erstmals mit ihrem Heißluftballon aufstiegen, der Physiker Charles, der im gleichen Jahr mit dem ersten Wasserstoffballon sich in die Lüfte erhob, stiegen noch nicht hoch genug auf, um die Höhenwirkung zu verspüren. Aber schon 1785 will Blanchard große Höhen mit einem Ballon erreicht haben und schreibt darüber :

«La nature languissait, j'éprouvais un engourdissement, prêlude d'un sommeil dangereux, lorsque, me levant malgré le peu de force qui me restait...» 
Dieser Bericht ist allerdings nicht unbedingt glaubwürdig.

Einwandfrei sind hingegen die Beobachtungen des Physikers RoBertson, der 1803 auf etwa $7000 \mathrm{~m}$ die ersten Höhenwirkungen an sich verspürte:

«A peine me trouvais-je dans cette atmosphère, que le malaise augmenta : j'étais dans une apathie morale et physique, nous pouvions à peine nous défendre du sommeil que nous redoutions comme la mort... A ce point élevé l'état où nous nous trouvions, était celui de l'indifférence... Là le physicien n'est plus sensible à la gloire et à la passion des découvertes: le danger même qui résoute dans ce voyage de la plus légère négligence ne l'occupe guère...»

Über das gleiche Erlebnis meint er an anderer Stelle:

«Lorsqu'on arrive à l'élévation de 3600 toises*, l'homme succombe par degré et d'une manière insensible à un sommeil lethargique : ses facultés morales s'éteignent longtemps avant ses facultés physiques. D'abord on n'a ni mémoire, ni soucis pour le présent et pour l'avenir. On oublie la surveillance qu'exige l'aérostat. Bientôt un sommeil lent et doux, auquel on sent l'impossibilité de résister, assoupit tous les membres et tient l'aéronaute dans une asphyxie complête, et sans doute mortelle si elle est prolongée...»

Noch größere Höhen erreichte Glaisher, der 1862 mit seinem Ballon ohne Sauerstoffatmung - wahrscheinlich auf $11000 \mathrm{~m}$ gelangte. Bei etwa $8800 \mathrm{~m}$ verlor er allerdings das Bewußtsein, nachdem eine Reihe klar erlebter neurologischer Ausfälle, wie Paresen und Sehstörungen, vorausgegangen waren.

Die Erscheinungen der Höhenkrankheit bei Ballonfahrern reizten auch den Dichter. In Stifters Condor werden während einer Ballonfahrt Schwindel, Blässe und Blutung aus den Lippen geschildert. E.A.PoE beschreibt in den Unvergleichlichen Abenteuern des Hans Pfaall die Höhenkrankheit wie folgt:

«Gegen sieben Uhr zeigte das Barometer eine Höhe von nicht weniger als neun und eine halbe Meile. Das Atemholen begann mir schwer zu werden. Auch der Kopf tat ungemein weh, und nachdem ich schon eine Zeitlang an den Wangen eine Feuchtigkeit verspürt hatte, entdeckte ich schließlich, daß es Blut war, das ziemlich stark aus meinen Ohren sickerte... Schon meinte ich, mir würden die Sinne schwinden.»

Auch eine Erklärung für diese Erscheinungen führt der Dichter an:

«Ihre Ursache war in der fortschreitenden Abnahme des gewohnten atmosphärischen Druckes auf die Körperoberfläche zu suchen ...»

1875 unternahm Tissandier mit Sivel und Croce-Spinelli den ersten größeren Aufstieg mit Sauerstoffausrüstung. Wegen ungenügender Mengen jedoch fanden Sivel und Croce-Spinelli auf etwa 8500 bis $8600 \mathrm{~m}$ Höhe den

$* 1$ toise $=1,949 \mathrm{~m}$. 
Tod. Daß aber bei gut funktionierender Sauerstoffversorgung diese einen wirksamen Schutz gegen die Höhenkrankheit darstellte, zeigten die Ballonaufstiege von Asmann und Berson (9155 m) sowie von Berson und Süring, die 1901 in die sonst tödliche Höhe von 11000 m gelangten. 1904 widmete Schrötter der Prophylaxe und Therapie der Luftdruckerkrankungen durch Sauerstoff eine eigene Untersuchung.

Die Bezeichnungen für die Bergkrankheit weisen zum Teil schon auf gewisse Ansichten über deren Ursache hin. Die peruanischen Indianer führten sie auf Dämpfe zurück, die von unterirdischen Erzadern wie z.B. Schwefelkies - Sorroche - herrührten. Auch die spanische Bezeichnung Veta - Erzgang - weist darauf hin. Im Himalaya erklären die Eingeborenen die Erscheinungen der Bergkrankheit mit Dünsten, die von giftigen Blumen ausgehen sollen (WEBB, zitiert bei Meyer-Ahrens, S. 29). Dem Missionar Huc zeigten die Eingeborenen auf einer Reise von der Mongolei nach Tibet ein feines Gras, das sie Pestdunst nannten. Cunningham (zitiert bei BERT), der die Bergkrankheit in Peru erlebte, führte dieselbe auf die elektrischen Verhältnisse in diesem Höhenklima zurück.

Obwohl sie keine giftigen Dünste der Erde oder Pflanzen annahmen, so waren auch Acosta und Ulloa der Ansicht, daß irgend etwas an der Gebirgsluft sie für die menschliche Atmung ungeeignet machte «sei es deren geringe Dichte oder eine andere unbekannte Eigenschaft»(Ulloa). Der geringen Dichte - «Subtilitate» - schrieben auch Fralichius sowie Borellus eine Rolle beim Zustandekommen der Bergkrankheit zu. Borellus brachte sie erstmals in Beziehung zur Muskelarbeit und stellte fest, daß erst bei körperlicher Anstrengung die Erscheinungen der Bergkrankheit auftraten. Seine Schlußfolgerungen aus dieser - allerdings nur für mittlere Höhen gültigen Beobachtung wurden weiter oben erwähnt. Auch später wurde immer wieder die Rolle der körperlichen Arbeit betont, und wiederholt wurde sogar die Ansicht geäußert, daß diesem Faktor allein die entscheidende Bedeutung zukomme (Bouger, Dufour [zitiert bei Fraenkel]). Auch in einer Zeit, als die experimentellen Untersuchungen über die Wirkung verminderten Luftdrucks von Boyle, Cigna, Bert u. a. längst bekannt waren, hörte man noch Ansichten wie diejenige, da $\beta$ die «sogenannte Bergkrankheit» meist auf Magenkatarrhe wegen der ungewohnten Gebirgskost zurückzuführen sei (Zsigmondy).

Die richtige Deutung der Ursachen der Bergkrankheit war aufs engste verbunden mit der Erforschung der Luft in ihren chemischen Eigenschaften 
und mit den experimentellen Untersuchungen über die Physiologie der Atmung. Langley gibt hierüber eine gute Übersicht, und Courville schildert die Anoxie in Geschichte, Legende und Folklore.

Schon Paracelsus (1493-1541) war der Ansicht, daß ebenso wie der Magen brauchbare Nahrung verwertet und unbrauchbare verwirft, die Lunge ihrerseits aus der Luft nur einen Teil verbrauche. Dieses «Lebenselixir» diene zur Wiederherstellung unserer Lebenskräfte. Boyle erwähnt diese Ansicht des Paracelsus (S. 139):

«Dicit quidem Paracelsus, quod uti ventriculi alimenta concoquit, partemque in usum corporis convertit, aliamque partem rejicit, ita Pulmo partem Aëris consumit, aliamque proscribit. Adeo ut juxta Hermeticum hunc Philosophum (sic enim secta illius eum compellari voluit) supponamus licet, aliquid in Aëre esse vitalis cuiusdam Elixiris (sit Verbo venia) quod refrigerandis restaurandisque vitalibus nostris spiritibus inserviat cui usui, cum crassior, et ultra comparationem major Aëris pars incommoda sit, minum videri non debet Animal prope indesinenter recentionis Aëris inspiratione indigere. Quamvis antem haec opinio non sit ... absurda, nihilominus ei assentire non debemus.»

Der holländische, in England lebende Physiker und «Mechaniker» Cornelis DrebBel (1572-1631) soll nach einem Bericht von Boyle bereits einiges über die Atmung gewußt oder geahnt haben. Drebbel sei der Ansicht gewesen, daß nur ein Teil der Luft für die Atmung diene, während der nicht verbrauchte, gewissermaßen der «cadaver aëris» nicht verwertbar sei. Da er ein Unterseeboot gebaut haben soll, hieß es auch, er habe dessen Insassen bei Durchfahrten durch die Themse mit einer chemischen Substanz versorgt, die er in Gefäßen mitnahm und die den Insassen des Bootes das Atmen in der verbrauchten Luft wieder erleichterte. Boyle schreibt hierüber wörtlich (S. 140):

«... putasse Drebellium non totum Aëris corpus, at certam illius Quintessentiam (ut loguntus chymici) seu sprituosam illius partem efficere, ut Respirationi inserviat, qua consumpta crassius quod reliquum est corpus, sive Cadaver (sit verbo venia) Aëris, vitalem flammam in corde residentem, fovere non valet : ita (in quantum colligere potui) praeter Mechanicum Navigii inventum, chymicum habuit Liquorem, quem praecipuum inter submarinae Navigationis Arcana, duxit. Quotiesamque enim puriorem Aëris partem consumptam vel nimium Respiratione depravatam, et eorum effluvii, qui navigarunt, saturatam animadvertit, racluso Vase illo Liquore completo, derepente turbato Aëri talem vitalium partium proportionem restituit, qualis efficere potuit, ut Respirationi aliqumdium subserviret, an dissipando vel praecipitando crassas exhalationes aliore intelligibili modo, jam non vacat in examen proferre: sufficiet mihi si addam mihi occasionem obvenisse praecipuis aliquot Amicis inserviendi, et cum hoc maxime conarer, ut cujusmodi stupendus hic Liquor esset expiscarer, fidenter affirmarunt Drebellium hoc nemini uspiam voluisse indicare, nec ex qua constaret materia, nisi unico tantum ostendere, qui me de ipsa re veritate fecit certiorem.» 
Boyles zahlreiche Experimente in einer Unterdruckglocke zeigten ihm schon etwa 1660 unter anderem, daß ein Tier in jenem Augenblick zu leben aufhörte, in dem eine brennende Kerze erlosch. Er folgerte daraus, « daß vielleicht in der Luft etwas sei, das wir noch nicht so recht verstehen, und das sie für das Leben der Tiere so notwendig mache». Mit Bezug auf die Bergkrankheit, deren Beschreibung durch Acosta er kannte, warf er die Frage auf, ob diese nicht darauf zurückzuführen sei, daß beim geringen Luftdruck im Blute sich Gasblasen bildeten, die mechanisch im Gefäßsystem Störungen bewirkten.

Cigna hatte etwa hundert Jahre später bemerkt, daß die Versuchstiere von Boyle bei einem Druck gestorben waren, der einer geringeren Höhe entsprach, als jene, welche von Menschen erstiegen wurde. Er beobachtete, $\mathrm{da}$ ß seine eigenen Versuchstiere länger lebten, wenn er sie in größeren Kammern dem gleichen Unterdruck aussetzte, oder wenn er die Kammern dauernd ventilierte. Diese Feststellung deutete er dahingehend, daß die Versuchstiere schädliche Dämpfe ausatmeten, mit denen die Kammer schließlich gesättigt wurde, und die dann zum Tode der Versuchstiere führten.

Zwar gelang es Priestley 1774 den Sauerstoff im Laboratorium darzustellen. Man begann aber erst später mit diesem Stoff als Faktor bei der Atmung zu rechnen. Der Genfer De Saussure, dessen Besteigung des Mont Blanc im August 1787 berühmter ist als die Erstbesteigung durch Jacques Balmat ein Jahr vorher, führte die Bergkrankheit auf einen Überschuß an Kohlensäure zurück. Er war der Auffassung, daß wegen der verdünnten Luft, Atmung und Zirkulation beschleunigt würden. Andererseits diskutierte er auch die Ansicht Hallers, daß durch den niederen Barometerstand der auf der Körperoberfläche lastende Druck sinke, und infolgedessen das Blut an die Oberfläche ströme. Dadurch werde in den inneren Organen und vor allem im Gehirn ein Zustand der Anämie erzeugt.

Alexander yon Humboldt dürfte der erste gewesen sein, der dem Problem der Bergkrankheit streng wissenschaftlich zu Leibe rückte. Während seiner Bergbesteigungen in den Anden maß er nicht nur den Luftdruck, sondern er untersuchte auch Luftproben auf ihren Sauerstoffgehalt. In seinem Brief an Delambre vom 25. November 1802 schildert er die Besteigung des $5300 \mathrm{~m}$ hohen Chimborazo und sagt u.a.

\footnotetext{
«... nous sentîmes tous un malaise, une débilité, une envie de vomir qui certainement provient du manque d'oxygène de ces régions, plus que de la rareté de l'air. Je n'ai trouvé que 0,20 d'oxygène à cette immense hauteur.»
} 
Er hatte also wohl als erster die Rolle des Sauerstoffmangels beim $\mathrm{Zu}$ standekommen der Höhenkrankheit erkannt.

Auch Chissold machte 1823 eine Verminderung des Sauerstoffgehaltes der Atmosphäre für die Erscheinungen der Bergkrankheit verantwortlich:

«. . . on account of the increased circulation of the blood, its carbon requires an increased quantity of oxygen of which only a diminished portion is necessarily present by the air in account of its diminished tenuity.»

Später wurde diese Ansicht immer häufiger vertreten, so z.B. durch Meyer-Ahrens. Dieser meint z. B. auf S. 132:

«Wenn nun aber das Auftreten der Bergkrankheit an mehr oder minder bedeutende Höhen gebunden ist, so fragt es sich, welches die Momente sind, die, von der Höhe abhängig, im Stande sind, solche Erscheinungen, wie sie die Bergkrankheit bilden, hervorzurufen. Und da spielen nach meiner Ansicht nun die Hauptrolle die absolute Abnahme des Sauerstoffgehaltes in der dünnern Luft, die raschere und quantitativ bedeutendere Wasserverdampfung und die intensive Einwirkung des vom Schnee zurückgeworfenen und direct auffallenden Lichtes, während ich dagegen in ätiologischer Beziehung der directen Einwirkung des verminderten Luftdruckes nur einen untergeordneten Rang einräumen möchte. Die nächste Ursache aber der Bergkrankheit suche ich in der Folge der absoluten Abnahme der Sauerstoffmenge und der starken Wasserverdampfung, gestörten Blutumwandlung, Blutbildung und veränderten Blutmischung und einer gleichzeitigen, durch die Einwirkung des Lichtes bedingten Störung der Gehirnfunction, welche ihrerseits wiederum auf den Blutbildungs- und Blutumwandlungsprozeß zurückwirken kann.»

Obwohl auch andere Kenner der Bergwelt, wie z.B. der englische Himalayaforscher Conway, die Bergkrankheit auf Sauerstoffmangel zurückführten, wurde diese Ansicht noch lange nicht von allen rückhaltlos akzeptiert. Sogar Mosso, der grundlegende physiologische Untersuchungen auf dem Monte Rosa durchführte, konnte sich nicht hierfür entscheiden, sondern vertrat noch um die Jahrhundertwende die Ansicht, daß eine Herabsetzung des $\mathrm{CO}_{2}$-Gehaltes im Blute die Hauptursache der Störungen sei. Etwa zur gleichen Zeit war Kronecker der Meinung, daß die Bergkrankheit verursacht sei «durch Stauungen im Lungenkreislauf infolge verminderten Luftdruckes».

Noch 1851 versuchte Payerne (zitiert nach Langley) durch eine Rechnung - die nur dem absoluten Sauerstoffgehalt, nicht aber dem Partialdruck Rechnung trug - die Unhaltbarkeit der Sauerstoffmangeltheorie nachzuweisen. Unter anderem hatte schon Jourdanet auf den Unterschied in der Sauerstoffsättigung hingewiesen, der bei beliebig langem Schütteln von Blut mit Luft in vitro, und der kurzen Kontaktzeit in der Lunge bestehe (zitiert bei Fraenkel). 
Die klassischen Untersuchungen des französischen Physiologen PAUL BERT, die in seinem Buch La pression barométrique (1878) niedergelegt sind, führten ihn zum klaren Ergebnis, daß der verminderte Luftdruck auf Lebewesen einzig durch Verminderung des Sauerstoffgehaltes von Luft, Blut und Gewebe wirke. Die Probe aufs Exempel gelang ihm, indem er 1874 in der Unterdruckkammer selber Sauerstoff atmete, und dessen wohltuende Wirkung auf die durch den Unterdruck bewirkten Erscheinungen beobachtete. Im gleichen Jahr noch wendeten zwei Ballonfahrer bei einem Aufstieg auf $7300 \mathrm{~m}$ erstmals Sauerstoffatmung an. Den tragischen Ausgang des ersten größeren Ballonaufstiegs im Jahre 1875 mit einer ungenügenden Sauerstoffmenge erwähnten wir bereits.

Mit den Arbeiten von Bert war die Frage nach den Ursachen der Höhenkrankheit in großen Zügen geklärt. Wie gezeigt werden konnte, hat bei der wissenschaftlichen Erforschung der Höhenkrankheit die Unterdruckkammer eine wichtige Rolle gespielt. Boyle hatte schon 1659 seine Tierversuche in der "machina pneumatica», einer Glocke, in der er durch Auspumpen einen Unterdruck erzeugte, veröffentlicht. Auch Cigna arbeitete hundert Jahre später mit ähnlicher Versuchsanordnung. Bert war 1874 der erste, der Selbstversuche in einer Unterdruckkammer machte, und - wie erwähnt - auch erstmals Sauerstoffatmung anwendete. Später erschienen die klassischen Untersuchungen von BARCROFT - der u.a. selber während sechs Tagen in einer Unterdruckkammer lebte - und die Selbstversuche von Haldane.

Die Forschung beschäftigte sich nun mit den physiologischen Grundlagen der Atmung und andererseits mit den experimentellen Untersuchungen über die physiologische und psychologische Wirkung des Sauerstoffmangels. Es sind hier u.a. die grundlegenden Untersuchungen von Haldane, von Loewy und von Barcroft zu nennen, dann aber auch die Ergebnisse zahlreicher Forschungsexpeditionen im Hochgebirge (Zuntz, Mosso usw.).

Durch den Aufschwung der Luftfahrt erfuhr die Erforschung der Sauerstoffmangelerscheinungen erneuten Auftrieb, besonders auch das Studium der psychischen Reaktionen beim Menschen. Während gelegentliche Beobachtungen über das psychische Verhalten in großen Höhen schon früher anläßlich von Hochgebirgswanderungen gemacht wurden (Humboldt, Meyer-Ahrens, Kronecker, Mosso, Reichel, Zuntz), wurden später auch systematische Untersuchungen, so z.B. von KuHN auf dem Jungfraujoch vorgenommen. Auch psychologische Testmethoden in der Unterdruckkam- 
mer, wie sie als erster wohl Barcroft durchführte, sind im Zusammenhang mit fliegerärztlichen Problemen in neuerer Zeit zahlreich angewandt worden (BInet, Hardmeier und Knoepfel, Jongbloed, MalméJac, Mumenthaler, v. Tavel, Wespi und Wissler). Auch durch das Atmen von Gasgemischen wurden die Wirkungen des Sauerstoffmangels auf die psychischen Funktionen geprüft (Dunlap, Gellhorn, Goralewski und Kraines) und unsere Kenntnisse auf diesem Gebiet wurden wesentlich erweitert*

* Herrn Dr. E. Hardmeier und Herrn Dr. H. K. Knoepfel möchte ich an dieser Stelle für zahlreiche Anregungen bestens danken.

\section{Literaturverzeichnis}

J. DE Acosta, Historia natural y moral de las Indias: en que se trata de cosas notables del Cielo, de los elementos, metales, plantas y animales, etc., Sevilla 1590, Neudruck Madrid 1894.

R. Asmann und A. Berson, Wissenschaftliche Luftfahrten, Braunschweig 1900 (zitiert nach KronecKer).

J. Barcroft, The Respiratory Function of the Blood, part I: Lessons from High altitude, Cambridge 1925.

A. Berson und Süring, Illustrierte aeronautische Mitteilungen 1901 (zitiert nach Zuntz, 1906).

P. BERT, Leçons sur la physiologie comparée de la respiration, Baillière, Paris 1870.

P. Bert, La pression barométrique. Recherches de physiologie expérimentale, Masson, Paris 1878.

L. Binet und V. Strumza, Hypoxie, hyperoxie et facultés intellectuelles de l'homme normal, Méd. Aéronautique 7 (1952) 516-8.

BLANChaRd, Relation du seizième voyage aérien de M. Blanchard, Gand 1786 (zitiert nach BERT).

J. A. Borellus, De motu animalium, Rom 1680, 1685.

Bouger, Relation abrégée du voyage fait au Pérou, etc. ..., Mém. Acad. des sci. de Paris 46 (1744) 249-97 (zitiert nach BERT).

R. Boyle, Nova experimenta physico-mechanica de vi aeris elastica et eiusdem effectibus, facta maximam partem in nova machina pneumatica, Genf 1677, in opera varia, Vol. I.

C. G. Bruce, The Assault on Mount Everest, London 1923.

G. F. Cigna, De causa extinctionis flammae, et animalium in aere interclusorum, Mél. phil. Math. Soc. Roy., Turin (1760) 168-203 (zitiert nach LANGLEY).

F. Cuissold, Narrative of an Ascent to the Summit of Mont Blanc, August $18^{\text {th }} 1822$. With an appendix upon the sensations experienced at great elevations, London 1823.

W. M. Conway, Climbing and Exploration in the Karakoram Himalayas, London 1884.

C. B. Courville, Contributions to the Study of Cerebral Anoxia, Bull. Los Angeles Neurol. Soc. (1950) 15 99-195. 
E. Döppig, Reise in Chile, Peru und am Amazonenstrom, Leipzig 1836.

C. G. Douglas, J. S. Haldane, Y. Henderson und E. C. Schneider, Physiological Observations Made on Pike's Peak Colorado, with Special Reference to Adaptation to Low Barometric Pressure, Trans. Roy. Soc. B 203 (1913) 185.

K. DunlaP, Medical Studies in Aviation, IV: Psychologic observations and methods, JAMA 71 (1918) 1392-3.

A. Durig et al., Physiologische Ergebnisse der im Jahre 1906 durchgeführten Monte-RosaExpedition, Denkschr. kaiserl. Akad. Wissenschaft. Mathem. Naturwiss. Klasse, 86. Band, Wien 1911.

E. A. Fitz-Gerald, The Highest Andes (zitiert nach Zuntz).

D. Fralichius, Geograph. gener. lib. i c 19 (zitiert nach Boyle).

A. Fraenkel und J. Geppert, Über die Wirkung der verdünnten Luft auf den Organismus. Eine Experimental-Untersuchung, Berlin 1883.

E. Gellhorn und S. H. Kraines, Word Associations as Affected by Deficient Oxygen, Excess of Carbon Dioxide and Hyperpnea, Arch. Neurol. and Psychiatry 38, (1937) 491-504.

E. Gellhorn, The Influence of Carbon Dioxide in Combatting the Effect of Oxygen Deficiency on Psychic Processes with Remarks on the Fundamental Relationship between Psychic and Physiologic Reactions, Amer. J. Psychiatry 93 (1937) 14.13-34.

J. Glaisher, C. Flamimarion, W. de Fonvielle und G. Tissandier, Voyages aériens, Paris 1870.

G. Goralewski, Anoxaemie und Zentralnervensystem, Z. ges. Neurol. et Psychiatrie 158 (1937) 3-8.

Güssfeldt, Reise in den Andes von Chile und Argentinien, Berlin 1888 (zitiert nach KronECKER).

J. S. Haldane, A. M. Kellas und E. L. Kennaway, Experiments on Acclimatisation to Reduced Atmospheric Pressure, J.Physiol. 53 (1919/20) 181-206.

J. S. Haldane, Respiration, New Haven 1922.

E. Hardmeier und H. K. Knoepfel, Psychiatric Aspects of Hypoxic States, Sörtrjck or Meddelanden frän Flyg - och Navalmedicinska Nämden (Stockholm) 1956 Nr. 2.

Huc, Souvenirs d'un voyage dans la Tartarie, le Thibet et la Chine, auszugsweise in Cannstatts Jahresbericht für 1850 (zitiert nach ZunTZ).

A. v. Humboldt, Correspondance scientifique et littéraire, Paris 1865.

J. Jongbloed, Über das psychische Verhalten während kurzen Aufenthalten auf 5000 Meter Höhe. Zugleich ein Beitrag zur Wirkung des Höhenklimas, Klin. Wschr. 14 (1935) 1564-8.

J. Jourdanet, Les altitudes de l'Amérique tropicale comparées au niveau des mers au point de vue de la constitution médicale, Paris 1861.

S. H. Kraines, The Correlation of Oxygen Deprivation with Intelligence, Constitution and Blood Pressure, Amer. J. Psychiatry 93 (1936/37) 1435-46.

H. KRONECKER, Die Bergkrankheit, Wien 1903.

Küchenmeister, Geschichtliche Darstellung der Lehre von dem Nutzen des Höhenklimas besonders für Phthisiker, Wiener med. Ztg. 1869 (zitiert nach ZunTz).

R. Kuнn, Bericht an den Chefarzt der Fl. und Flab Trp. über die Ergebnisse der psychologischen Untersuchungen auf Jungfraujoch vom 21.-27. August 1942, Manuskript. 
R. KuHN, Über die psychischen Wirkungen des Sauerstoffmangels. Vortrag vor der Versammlung der Schweizerischen Gesellschaft für Psychiatrie in Genf, 5. Dezember 1943, Manuskript.

L. L. Langley, Historical Introduction to Physiology of Anoxia, Bull. Hist. Med. 14 (1943) 321-40.

A. Loewy, Physiologie des Höhenklimas, Springer, Berlin 1932.

J. Malmejac, R. Ruffieux, G. Chardon und F. Serrell, Etude sur les premières manifestations psycho-physiologiques consécutives à l'anoxie, Rapport au Congr. Nat. Aviat. Franç. Paris 1945.

C. Meyer-Ahrens, Die Bergkrankheit, oder der Einflu $\beta$ des Ersteigens großer Höhen auf den thierischen Organismus, Leipzig 1854.

Middendorf, Das Hochland von Peru, Berlin 1895 (zitiert nach Zuntz).

Moorcroft, Asiatic Researches, T. XII, Calcutta 1816 (zitiert bei Meyer-Ahrens).

A. Mosso, Fisiologia dell'uomo sulle alpi, Milano 1897.

A. Mosso und Galeotti, L'action physiologique de l'alcool à des grandes altitudes, Turin 1904. (zitiert nach DuRIG).

M. Mumenthaler, Influence de l'hypoxie sur le résultat du test de Rorschach, Méd. Aéronautique 10 (1955) 31-6.

D'Orbigny, Voyage dans l'Amérique méridionale 1826-33, Paris 1839 (zitiert nach Zuntz).

J. Oudot, L'expédition française de l'Himalaya, Pr. méd. 58 (1950) 1000.

J. Oudot, Observations physiologiques et cliniques en haute montagne, Pr. méd. 59 (1951) 297-300.

A. Payot, Du mal des montagnes, Diss., Paris 1881.

R. Pfisterer, Die Dhaulagiri-Expedition des Akademischen Alpenclubs Zürich, Schweiz. med. Wschr. 84 (1954) 716-23.

E. A. PoE, Das unvergleichliche Abenteuer eines gewissen Hans Pfaall, übersetzt von GISELA ETZel, Wien 1947.

H. ReicheL, Ergebnisse der Monte Rosa-Expedition vom Jahre 1906. Über die Dauer einfacher psychischer Vorgänge unter dem Einfluß des Höhenklimas, und über die psychische Alkoholwirkung in großen Höhen, Denkschr. Kaiserl. Akad. Wiss. 86 (1911) 14-7 und 79-113.

Robertson, Journal de Paris, 16. Mai 1812 (zitiert nach Bert).

Robertson, Relation adressée au président de l'Acad. imp. de Saint-Pétersb., Mémoires récréatifs scientifiques et anecdotiques, Paris 1840 (zitiert nach BERT).

H. B. De SAussure, Voyages dans les alpes. Partie pittoresque des ouvrages de H. B. de Saussure, Neuchâtel 1803.

H. B. DE SAussure, La première ascension du Mont Blanc, Neuchâtel 1923.

A. und R.v. Schlagintweit, Results of a Scientific Mission to India and High Asia 1854-58, London 1861 (zitiert nach ZuNTZ).

H. v. Schrötter, Zur Kenntnis der Bergkrankheit, Wien 1899 (zitiert nach Durig).

H. v. Schвӧтter, Der Sauerstoff in Prophylaxe und Therapie der Luftdruckerkrankungen, Berlin 1904 (zitiert nach DuRIG).

A. Stifter, Der Condor.

F. v. Tavel, Die Auswirkungen des Sauerstoffmangels auf den menschlichen Organismus bei kurzfristigem Aufenthalt in großer Höhe, Helvetica Physiologica Acta, Suppl. I/1943. 
G. Tissandier, Le voyage à grande hauteur du Ballon «Le Zénith», La Nature 3 (1875) 337-44 (zitiert nach BERT).

J. J. Tschudi, Peru, Reiseskizzen aus den Jahren 1838-42, St. Gallen 1846.

A. D'UlloA, Mémoires philosophiques, historiques, physiques, concernant la découverte de l'Amérique etc., Traduction, Paris 1787.

H. WESPI, Ủber das psychische Verhalten bei kurzem Aufenthalt auf $5000 \mathrm{~m}$ Höhe, Klin. Wschr. 15 (1936) 701-2.

E. WhYMPer, Travels amongst the Grat Andes of the Equator, London 1892 (zitiert nach Zuntz).

H. Wissler und H. WeSPI, Versuche über die psychischen Insuffizienzerscheinungen bei vermindertem Luftdruck, Schweiz. med. Wschr. 63 (1933) 460.

E. Zsigmondy, Die Gefahren der Alpen, Leipzig 1888.

N. Zuntz und L. Zuntz, Über die Wirkung des Hochgebirges auf den menschlichen Organismus, Berlin 1897.

N. Zuntz, A. Loewy, F. Müller und W. Caspari, Höhenklima und Bergwanderungen in ihrer Wirkung auf den Menschen, Berlin 1906. 